\title{
Efeitos de um programa de ginástica laboral sobre indicadores de saúde e dor corporal em trabalhadores de um hospital de ensino do Rio Grande do Sul
}

\author{
Effects of an labor gymnastics program on health and body pain \\ indicators in workers of a Rio Grande do Sul teaching hospital
}

\author{
Leandro Tibiriçá Burgos, ${ }^{1}$ Cézane Priscila Reuter, ${ }^{1}$ Leticia Borfe, ${ }^{1}$ Miria Suzana Burgos
}

'Universidade de Santa Cruz do Sul (Unisc), Santa Cruz do Sul, RS, Brasil.

Recebido em: 11/12/2017 / Aceito em: 18/12/2017 / Publicado em: 30/12/2017

Iburgos@unisc.br

\section{RESUMO}

Objetivo: verificar se um programa de ginástica laboral (GL) promove efeitos sobre indicadores de saúde e presença de desconforto e dor musculoesquelética em trabalhadores de um hospital. Método: estudo semi-experimental realizado com 46 trabalhadores em atividade laboral regular, do sexo feminino, de um hospital de ensino do Rio Grande do Sul. Foram realizadas sessões estruturadas com exercícios funcionais, resistência muscular localizada e exercícios de alongamento e relaxamento, durante seis meses, três vezes por semana, com duração de 15 minutos cada. Antes e após o programa de GL, foram avaliados indicadores antropométricos (índice de massa corporal - IMC; circunferência da cintura - CC; relação cintura-quadril - RCQ e percentual de gordura corporal - \%G), nível de flexibilidade (teste do manguito rotador) e a percepção de desconforto e dor corporal. Resultados: foram observadas alterações significativas na CC $(p=0,014)$, na $\mathrm{RCQ}$ e nos níveis de flexibilidade do ombro direito $(p<0,001)$ e do ombro esquerdo $(p=0,004)$. Para CC, duas mulheres que possuíam "risco aumentado" passaram para a classificação "normal". No entanto, não foram observadas diferenças significativas sobre a percepção de dor corporal após a intervenção com a GL. Conclusão: o programa de GL promoveu efeitos em parâmetros antropométricos e de flexibilidade, mas não alterou a percepção de desconforto e dor corporal nos trabalhadores avaliados.

Palavras-chave: Ginástica Laboral; DORT; Saúde dos Trabalhadores.

\section{ABSTRACT}

Objective: to verify if a workout program (GL) promotes effects on health indicators and presence of musculoskeletal discomfort and pain in hospital workers. Method: a semi-experimental study was carried out with 46 workers in regular female work at a teaching hospital in Rio Grande do Sul. Structured sessions with functional exercises, localized muscular resistance and stretching and relaxation exercises were performed for six months, three times a week, lasting 15 minutes each. Before and after the GL program, anthropometric indicators (body mass index - BMI, waist circumference - WC, waist-hip ratio - WHR and percentage of body fat - $\%$ G), flexibility level (rotator cuff test) and the perception of discomfort and body pain. Results: significant changes were observed in the WC ( $p=0.014)$, the WHR and the levels of flexibility of the right shoulder $(p<0.001)$ and left shoulder ( $p=0.004)$. For CC, two women who had "increased risk" were classified as "normal". However, no significant differences were observed in the perception of body pain after the intervention with GL. Conclusion: the GL program promoted effects on anthropometric parameters and flexibility, but did not alter the perception of discomfort and body pain in the evaluated workers.

Keywords: Labor gymnastics; DORT; Workers' Health. 


\section{INTRODUÇÃO}

As grandes mudanças que ocorrem no mundo do trabalho, devido a inovações organizacionais e tecnológicas, exigem dos trabalhadores uma adaptação em ritmo muito elevado. ${ }^{1}$ Estas mudanças podem levar o trabalhador a circunstâncias indesejadas e críticas de desconforto, o que pode gerar mal estar, absenteísmo, que se refletem no bom andamento da Instituição, ${ }^{2}$ principalmente quando nos referimos a uma unidade hospitalar, que por si só, lida dia e noite, com problemas de saúde. Como consequências, destaca-se o cansaço e o estresse, que se refletem e se somatizam em diferentes partes do corpo, gerando dor e mal estar. ${ }^{3}$

A ginástica laboral (GL) surge, então, como ferramenta importante no processo de promoção da saúde do trabalhador, ${ }^{4}$ melhorando a qualidade de vida dos colaboradores institucionais, contribuindo na prevenção de Distúrbios Osteomioarticulares Relacionados ao Trabalho e Lesões por Esforços Repetitivos (DORT/LER), atuando nas estruturas anatômicas e fisiológicas, prevenindo e corrigindo alterações posturais, amenizando a dor muscular e reabilitando colaboradores já atingidos pelos distúrbios posturais ${ }^{1,5-9} \mathrm{~A}$ implantação de um programa de GL também propõe modificações benéficas nos recursos humanos e financeiros das empresas, aumentando a produtividade, diminuindo a incidência das doenças ocupacionais e despesas médicas. ${ }^{10,11}$

Diante do contexto apresentado, o presente estudo objetiva verificar se um programa de ginástica laboral promove efeitos sobre indicadores de saúde e presença de desconforto e dor musculoesquelética em trabalhadores de um hospital de ensino.

\section{MÉTODO}

Estudo semi-experimental realizado com 46 trabaIhadores em atividade laboral regular, do sexo feminino, de um hospital de ensino do Vale do Rio Pardo, Rio Grande do Sul. Todas as participantes assinaram um termo de consentimento livre e esclarecido, autorizando a participação na pesquisa.

Antes e após o programa de GL, foram avaliados indicadores antropométricos (índice de massa corporal IMC; circunferência da cintura - CC; relação cintura-quadril - RCQ e percentual de gordura corporal - \%G), nível de flexibilidade e a percepção de desconforto e dor corporal.

O IMC foi calculado utilizando-se a fórmula IMC = peso/altura ${ }^{2}\left(\mathrm{~kg} / \mathrm{m}^{2}\right)$ e classificado de acordo com os pontos de corte estabelecidos pela Organização Mundial da Saúde, ${ }^{12}$ em quatro categorias: baixo peso, eutrofia, sobrepeso e obesidade. A circunferência da cintura foi obtida utilizando como referência a parte mais estreita entre as costelas e a crista ilíaca, posteriormente classificada de acordo com critérios estabelecidos pela Organização Mundial da Saúde ${ }^{12}$ em duas classes: baixo risco, normal, risco aumentado e alto risco. A Relação Cintura/Quadril (RCQ) foi calculada através da razão entre a $\mathrm{CC}$ e a $\mathrm{CO}$, classificada em risco baixo, risco moderado, risco alto e risco muito alto. ${ }^{12}$ Para mensuração do percentual de gordura foi realizada a avaliação em três dobras cutâneas, obtidas por meio do compasso de Lange, estimado pela equação Jackson e Pollock e, posteriormente, de Siri' ${ }^{13}$ e classificado segundo Pollock e Wilmore. ${ }^{14}$

Para avaliar o nível de flexibilidade, foi utilizado o teste de extensão de ombros do manguito rotador, ${ }^{15}$ sendo classificado com baixa condição, abaixo da média, média, acima da média e superior ou atlético. A percepção de desconforto e dor corporal foi observada através do questionário adaptado do instrumento Br-MPO (questionário de dor McGill). ${ }^{16}$

Foram realizadas sessões estruturadas de GL durante seis meses, três vezes por semana, com duração de 15 minutos cada. As sessões foram compostas de exercícios funcionais, de resistência muscular localizada e exercícios de alongamento e relaxamento. Todas as sessões foram realizadas de forma sistematizada, prescritas por meio de microciclos, mesociclos e macrociclos, desenvolvidas especificamente em cada setor do hospital.

Utilizou-se o programa estatístico SPSS v. 20.0 (IBM, Chicago, EUA) para digitação e análise dos dados. Foi realizada a estatística descritiva (número e percentual) para caracterização da amostra nos momentos pré e pós-teste. Para comparar possíveis modificações entre estes dois momentos, para as variáveis categóricas ordinais, utilizou-se o teste pareado de Wilcoxon. Para variáveis dicotômicas, utilizou-se o teste de McNemar. Foram consideradas diferenças significativas para $p<0,05$.

Este estudo passou pelo Comitê de Ética em Pesquisa da Universidade de Santa Cruz do Sul (UNISC) e foi aprovado para realização. Os indivíduos participantes da pesquisa assinaram um termo de consentimento livre e esclarecido.

\section{RESULTADOS E DISCUSSÃO}

A tabela 1 apresenta os dados descritivos (frequência e percentual) dos indicadores de saúde avaliados no pré e pós-teste para os indicadores antropométricos (IMC, CC, RCO e \%G) e extensão de ombros (manguito rotador - ombro direito e esquerdo).

Tabela 1 - Dados descritivos dos indicadores de saúde avaliados no pré e pós-teste.

\begin{tabular}{|c|c|c|}
\hline & $\begin{array}{c}\text { Pré-teste }(N=46) \\
n(\%)\end{array}$ & $\begin{array}{c}\text { Pós-teste }(\mathrm{N}=46) \\
\mathrm{n}(\%)\end{array}$ \\
\hline \multicolumn{3}{|l|}{ IMC } \\
\hline Baixo peso & - & $1(2,2)$ \\
\hline Eutrofia & $23(50,0)$ & $22(47,8)$ \\
\hline Sobrepeso & $19(41,3)$ & $19(41,3)$ \\
\hline Obesidade & $4(8,7)$ & $4(8,7)$ \\
\hline \multicolumn{3}{|l|}{$\mathrm{CC}$} \\
\hline Baixo risco & - & $1(2,2)$ \\
\hline Normal & $22(47,8)$ & $23(50,0)$ \\
\hline Risco aumentado & $13(28,3)$ & $14(30,4)$ \\
\hline Alto risco & $11(23,9)$ & $8(17,4)$ \\
\hline \multicolumn{3}{|l|}{$\mathrm{RCO}$} \\
\hline Risco baixo & $7(15,2)$ & $2(4,3)$ \\
\hline Risco moderado & $15(32,6)$ & $16(34,8)$ \\
\hline Risco alto & $16(34,8)$ & $17(37,0)$ \\
\hline Risco muito alto & $8(17,4)$ & $11(23,9)$ \\
\hline \multicolumn{3}{|l|}{$\% G$} \\
\hline Muito ruim & $13(28,3)$ & $15(32,6)$ \\
\hline Ruim & $14(30,4)$ & $14(30,4)$ \\
\hline Abaixo da média & $12(26,1)$ & $8(17,4)$ \\
\hline
\end{tabular}




\begin{tabular}{lcc}
\hline Média & $6(13,0)$ & $8(17,4)$ \\
Acima da média & $1(2,2)$ & $1(2,2)$ \\
Manguito rotador - Ombro direito & \\
Baixa condição & $25(54,3)$ & $15(32,6)$ \\
Abaixo da média & $11(23,9)$ & $13(28,3)$ \\
Média & $4(8,7)$ & $6(13,0)$ \\
Acima da média & $1(2,2)$ & - \\
Superior ou atlético & $5(10,9)$ & $12(26,1)$ \\
Manguito rotador - Ombro esquerdo & \\
Baixa condição & $21(45,6)$ & $19(41,3)$ \\
Abaixo da média & $5(10,9)$ & $1(2,2)$ \\
Média & $13(28,3)$ & $13(28,3)$ \\
Acima da média & $3(6,5)$ & $3(6,5)$ \\
Superior ou atlético & $4(8,7)$ & $10(21,7)$ \\
\hline
\end{tabular}

IMC: índice de massa corporal; CC: circunferência da cintura; RCQ: relação cinturaquadril; \% G: percentual de gordura.

A tabela 2 compara os indicadores de saúde nos momentos pré e pós-teste. Observam-se, após a intervenção com a ginástica laboral, alterações significativas na $C C(p=0,014), R C Q$, manguito rotador - ombro direito $(p<0,001)$ e ombro esquerdo $(p=0,004)$. Para CC, duas mulheres que possuíam "risco aumentado" passaram para a classificação "normal". Os demais indicadores antropométricos não apresentaram melhora.
Estudo realizado em Portugal, com trabalhadores de uma indústria multinacional, demonstrou que o programa de intervenção com GL, durante seis meses, não diminuiu os indicadores antropométricos avaliados (peso, IMC, circunferência da cintura e \%G),${ }^{17}$ corroborando os dados do presente estudo, excetuando-se os resultados obtidos para a CC.

Ainda na tabela 2, observa-se que houve melhora na extensão de ombros, avaliada pelo manguito rotador. Para o ombro direito, duas mulheres que estavam na classe "baixa condição" e três que estavam "abaixo da média" passaram para a classificação "média". Da mesma forma com o ombro esquerdo, duas mulheres que estavam na classe "baixa condição" e duas que estavam "abaixo da média" passaram para a classificação "média". Salienta-se, ainda, que das mulheres que estavam na classificação "média", três passaram para a classificação "superior ou atlético", tanto para o ombro direito, quanto para o ombro esquerdo. Em mulheres trabalhadoras de uma indústria da Espanha, um programa de GL foi efetivo na melhora da extensão dos músculos após 12 semanas. ${ }^{18}$ Em trabalhadores coreanos, um programa de GL realizado durante 12 semanas foi efetivo na melhora da flexibilidade; porém, não demonstrou resultados satisfatórios para força muscular. ${ }^{19}$

Tabela 2 - Comparação dos indicadores de saúde no pré e pós-teste ( $N=46)$.

\begin{tabular}{|c|c|c|c|c|c|c|c|}
\hline \multicolumn{8}{|c|}{ IMC pós-teste } \\
\hline IMC pré-teste & Baixo peso & Recomendável & & Sobrepeso & & Obesidade I & $p$ \\
\hline Recomendável & 1 & 22 & & 0 & & 0 & 0,317 \\
\hline Sobrepeso & 0 & 0 & & 19 & & 0 & \\
\hline Obesidade I & 0 & 0 & & 0 & & 4 & \\
\hline \multicolumn{8}{|c|}{ CC pós-teste } \\
\hline CC pré-teste & Baixo risco & Normal & Risc & o aument & & Alto risco & $p$ \\
\hline Normal & 1 & 21 & & 0 & & 0 & 0,014 \\
\hline Risco aumentado & 0 & 2 & & 11 & & 0 & \\
\hline Alto risco & 0 & 0 & & 3 & & 8 & \\
\hline \multicolumn{8}{|c|}{ RCQ pós-teste } \\
\hline RCQ pré-teste & Baixo risco & Risco moderado & & Risco alto & & Risco muito alto & $p$ \\
\hline Risco baixo & 2 & 3 & & 1 & & 1 & 0,040 \\
\hline Risco moderado & 0 & 9 & & 5 & & 1 & \\
\hline Risco alto & 0 & 4 & & 9 & & 3 & \\
\hline Risco muito alto & 0 & 0 & & 2 & & 6 & \\
\hline \multicolumn{8}{|c|}{ \%G pós-teste } \\
\hline$\% G$ pré-teste & Muito ruim & Ruim & Abaixo da & a média & Média & Acima da Média & $p$ \\
\hline Muito ruim & 12 & 1 & 0 & & 0 & 0 & 0,617 \\
\hline Ruim & 3 & 10 & 1 & & 0 & 0 & \\
\hline Abaixo da média & 0 & 3 & 6 & & 2 & 1 & \\
\hline Média & 0 & 0 & 1 & & 5 & 0 & \\
\hline Acima da média & 0 & 0 & 0 & & 1 & 0 & \\
\hline \multicolumn{8}{|c|}{ MR - OD pós-teste } \\
\hline MR - OD pré-teste & Baixa condição & Abaixo da média & & Média & & Superior ou atlético & $p$ \\
\hline Baixa condição & 15 & 7 & & 2 & & 1 & $<0,001$ \\
\hline Abaixo da média & 0 & 6 & & 3 & & 2 & \\
\hline Média & 0 & 0 & & 1 & & 3 & \\
\hline Acima da média & 0 & 0 & & 0 & & 1 & \\
\hline Superior ou atlético & 0 & 0 & & 0 & & 5 & \\
\hline \multicolumn{8}{|c|}{ MR - OE pós-teste } \\
\hline MR - OE pré-teste & Baixa condição & Abaixo da média & Média & Acima & a média & Superior ou atlético & $p$ \\
\hline Baixa condição & 17 & 1 & 2 & & & 0 & 0,004 \\
\hline Abaixo da média & 2 & 0 & 2 & & 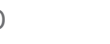 & 1 & \\
\hline Média & 0 & 0 & 8 & & 2 & 3 & \\
\hline Acima da média & 0 & 0 & 1 & & 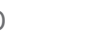 & 2 & \\
\hline Superior ou atlético & 0 & 0 & 0 & & ) & 4 & \\
\hline
\end{tabular}

IMC: índice de massa corporal; CC: circunferência da cintura; RCQ: relação cintura-quadril; \% G: percentual de gordura; MR - OD: manguito rotador - ombro direito; $M R$ - OE: manguito rotador - ombro esquerdo; diferença significativa para $p<0,05$; teste de Wilcoxon. 
A tabela 3 apresenta a presença de desconforto e dor no pescoço, costas e bacia, nos momentos pré e pós-teste. Não foram observadas diferenças significativas após a intervenção com a GL. Estudo realizado com funcionárias de uma universidade de Umuarama, no Paraná, demonstrou que $75 \%$ destas apresentaram melhora na sintomatologia da dor após seis meses de um programa de $\mathrm{GL}$, principalmente na coluna dorsal, a qual teve uma redução de $19 \%$ na presença de dor. ${ }^{20}$ Estudo realizado com funcionários de outra universidade, localizada em São Leopoldo, RS, demonstrou que um programa de GL reduziu a dor das costas destes sujeitos após 36 meses. ${ }^{21}$ Em funcionários de uma indústria multinacional localizada em Portugal, seis meses de um programa de GL foi efetivo na melhora de dor na região dorsal. ${ }^{17}$

Para os membros superiores (Tabela 4), também não foram encontradas diferenças significativas após a intervenção da GL, na presença de desconforto ou dor. Estudo realizado com funcionários de um laboratório farmacêutico de Irati, no Paraná, demonstrou que um programa de $G L$, realizado por três meses, foi efetivo na melhora da sensação de dor, reduzindo as reclamações em 76\%; por outro lado, permaneceram as dores nos punhos, devido à atividade desempenhada no laboratório (manipulação de medicamentos). ${ }^{22}$ Em trabalhadores portugueses, houve melhora na dor dos cotovelos após seis meses de intervenção com GL. ${ }^{17}$

A tabela 5 demonstra que a presença de desconforto e dor nos membros inferiores não diferiu significativamente após a intervenção. Com resultados diferentes, estudo realizado com trabalhadores do setor administrativo de Portão, no Rio Grande do Sul demonstrou melhora das intensidades mais altas de dor de todos dos segmentos, inclusive das pernas, após os três meses do programa de GL. ${ }^{1}$

Além da redução da dor e melhora da postura, ${ }^{9}$ a implantação da GL proporciona benefícios aos trabalhadores que auxiliam nas atividades laborativas, redução nos afastamentos do trabalho e melhora na qualidade de vida deste trabalhador. Além disso, contribui na melhora dos aspectos físicos, sociais, psicológicos e consequente aumento na produção devido sua motivação. ${ }^{23}$

Tabela 3 - Presença de desconforto e dor no pescoço, costas e bacia ( $N=21)$.

\begin{tabular}{|c|c|c|c|c|c|c|}
\hline & \multirow{3}{*}{ Pré-teste } & \multicolumn{4}{|c|}{ Pós-teste } & \multirow{3}{*}{$p^{*}$} \\
\hline & & \multicolumn{2}{|c|}{ Desconforto } & \multicolumn{2}{|c|}{ Dor } & \\
\hline & & Não & Sim & Não & Sim & \\
\hline \multirow{2}{*}{ Desconforto Pescoço } & Não & 6 & 4 & 6 & 4 & 1,000 \\
\hline & Sim & 5 & 6 & 5 & 6 & \\
\hline \multirow[t]{2}{*}{ Dor Pescoço } & Não & - & - & - & - & 1,000 \\
\hline & Sim & - & - & - & - & \\
\hline \multirow[t]{2}{*}{ Desconforto costas superior } & Não & 8 & 5 & 8 & 5 & 1,000 \\
\hline & Sim & 6 & 2 & 6 & 2 & \\
\hline \multirow[t]{2}{*}{ Dor costas superior } & Não & - & - & - & - & 0,453 \\
\hline & Sim & - & - & - & - & \\
\hline \multirow[t]{2}{*}{ Desconforto costas média } & Não & 9 & 4 & 9 & 4 & 1,000 \\
\hline & Sim & 5 & 3 & 5 & 3 & \\
\hline \multirow[t]{2}{*}{ Dor costas média } & Não & - & - & - & - & 0,375 \\
\hline & Sim & - & - & - & - & \\
\hline \multirow[t]{2}{*}{ Desconforto costas inferior } & Não & 13 & 4 & 13 & 4 & 0,375 \\
\hline & Sim & 1 & 3 & 1 & 3 & \\
\hline \multirow[t]{2}{*}{ Dor costas inferior } & Não & - & - & - & - & 0,070 \\
\hline & Sim & - & - & - & - & \\
\hline \multirow[t]{2}{*}{ Desconforto bacia } & Não & 14 & 5 & 14 & 5 & 0,219 \\
\hline & Sim & 1 & 1 & 1 & 1 & \\
\hline \multirow[t]{2}{*}{ Dor bacia } & Não & - & - & - & - & 1,000 \\
\hline & Sim & - & - & - & - & \\
\hline
\end{tabular}

Dados apresentados em frequência; *Teste de McNemar

Tabela 4 - Presença de desconforto e dor nos membros superiores ( $N=21)$.

\begin{tabular}{|c|c|c|c|c|c|c|}
\hline & \multirow{3}{*}{ Pré-teste } & \multicolumn{4}{|c|}{ Pós-teste } & \multirow{3}{*}{$p^{*}$} \\
\hline & & \multicolumn{2}{|c|}{ Desconforto } & \multicolumn{2}{|c|}{ Dor } & \\
\hline & & Não & Sim & Não & Sim & \\
\hline \multirow[t]{2}{*}{ Desconforto ombro direito } & Não & 9 & 5 & - & - & 1,000 \\
\hline & Sim & 5 & 2 & - & - & \\
\hline \multirow[t]{2}{*}{ Dor ombro direito } & Não & - & - & 13 & 5 & 0,063 \\
\hline & Sim & - & - & 0 & 3 & \\
\hline \multirow[t]{2}{*}{ Desconforto ombro esquerdo } & Não & 7 & 7 & - & - & 0,549 \\
\hline & Sim & 4 & 3 & - & - & \\
\hline \multirow[t]{2}{*}{ Dor ombro esquerdo } & Não & - & - & 13 & 3 & 1,000 \\
\hline & Sim & - & - & 2 & 3 & \\
\hline \multirow[t]{2}{*}{ Desconforto braço direito } & Não & 13 & 2 & - & - & 1,000 \\
\hline & Sim & 3 & 3 & - & - & \\
\hline \multirow[t]{2}{*}{ Dor braço direito } & Não & - & - & 18 & 2 & 0,500 \\
\hline & Sim & - & - & 0 & 1 & \\
\hline
\end{tabular}


EFEITOS DE UM PROGRAMA DE GINÁSTICA LABORAL SOBRE INDICADORES DE SAÚDE E DOR CORPORAL EM TRABALHADORES DE UM HOSPITAL DE ENSINO DO RIO GRANDE DO SUL Leandro Tibiriçá Burgos, Cézane Priscila Reuter, Leticio Borfe. Miria Suzana Burgos.

\begin{tabular}{|c|c|c|c|c|c|c|}
\hline \multirow[t]{2}{*}{ Desconforto braço esquerdo } & Não & 12 & 4 & - & - & 0,687 \\
\hline & Sim & 2 & 3 & - & - & \\
\hline \multirow{2}{*}{ Dor braço esquerdo } & Não & - & - & 21 & 0 & 1,000 \\
\hline & Sim & - & - & 0 & 0 & \\
\hline \multirow[t]{2}{*}{ Desconforto cotovelo direito } & Não & 14 & 4 & - & - & 0,125 \\
\hline & Sim & 0 & 3 & - & - & \\
\hline \multirow[t]{2}{*}{ Dor cotovelo direito } & Não & - & - & 21 & 0 & 1,000 \\
\hline & Sim & - & - & 0 & 0 & \\
\hline \multirow[t]{2}{*}{ Desconforto cotovelo esquerdo } & Não & 14 & 5 & - & - & 0,063 \\
\hline & Sim & 0 & 2 & - & & \\
\hline \multirow[t]{2}{*}{ Dor cotovelo esquerdo } & Não & - & - & 21 & 0 & 1,000 \\
\hline & Sim & - & - & 0 & 0 & \\
\hline \multirow[t]{2}{*}{ Desconforto antebraço direito } & Não & 14 & 4 & - & - & 0,375 \\
\hline & Sim & 1 & 2 & - & - & \\
\hline \multirow[t]{2}{*}{ Dor antebraço direito } & Não & - & - & 19 & 1 & 1,000 \\
\hline & Sim & - & - & 1 & 0 & \\
\hline \multirow[t]{2}{*}{ Desconforto antebraço esquerdo } & Não & 13 & 4 & - & - & 0,687 \\
\hline & Sim & 2 & 2 & - & - & \\
\hline \multirow[t]{2}{*}{ Dor antebraço esquerdo } & Não & - & - & 21 & 0 & 1,000 \\
\hline & Sim & - & - & 0 & 0 & \\
\hline \multirow[t]{2}{*}{ Desconforto punho direito } & Não & 15 & 4 & - & - & 0,375 \\
\hline & Sim & 1 & 1 & - & - & \\
\hline \multirow[t]{2}{*}{ Dor punho direito } & Não & - & - & 16 & 2 & 0,500 \\
\hline & Sim & - & - & 0 & 3 & \\
\hline \multirow[t]{2}{*}{ Desconforto punho esquerdo } & Não & 12 & 4 & - & - & 1,000 \\
\hline & Sim & 3 & 2 & - & - & \\
\hline \multirow[t]{2}{*}{ Dor punho esquerdo } & Não & - & - & 20 & 0 & 1,000 \\
\hline & Sim & - & - & 0 & 1 & \\
\hline \multirow[t]{2}{*}{ Desconforto mão direita } & Não & 15 & 4 & - & - & 0,375 \\
\hline & Sim & 1 & 1 & - & - & \\
\hline \multirow[t]{2}{*}{ Dor mão direita } & Não & - & - & 18 & 1 & 1,000 \\
\hline & Sim & - & - & 1 & 1 & \\
\hline \multirow[t]{2}{*}{ Desconforto mão esquerda } & Não & 14 & 4 & - & - & 0,687 \\
\hline & Sim & 2 & 1 & - & - & \\
\hline \multirow[t]{2}{*}{ Dor mão esquerda } & Não & - & - & 20 & 1 & 1,000 \\
\hline & Sim & - & - & 0 & 0 & \\
\hline
\end{tabular}

Dados apresentados em frequência; * Teste de McNemar

Tabela 5 - Presença de desconforto e dor nos membros inferiores ( $N=21$ ).

\begin{tabular}{|c|c|c|c|c|c|c|}
\hline & \multirow{3}{*}{ Pré-teste } & \multicolumn{4}{|c|}{ Pós-teste } & \multirow{3}{*}{$\mathrm{p}^{*}$} \\
\hline & & \multicolumn{2}{|c|}{ Desconforto } & \multicolumn{2}{|c|}{ Dor } & \\
\hline & & Não & Sim & Não & Sim & \\
\hline \multirow{2}{*}{ Desconforto coxa direita } & Não & 13 & 5 & - & - & 0,063 \\
\hline & Sim & 0 & 3 & - & - & \\
\hline \multirow[t]{2}{*}{ Dor coxa direita } & Não & - & - & 21 & 0 & 1,000 \\
\hline & Sim & - & - & 0 & 0 & \\
\hline \multirow{2}{*}{ Desconforto coxa esquerda } & Não & 13 & 5 & - & - & 0,063 \\
\hline & Sim & 0 & 3 & - & - & \\
\hline \multirow[t]{2}{*}{ Dor coxa esquerda } & Não & - & - & 21 & 0 & 1,000 \\
\hline & Sim & - & - & 0 & 0 & \\
\hline \multirow[t]{2}{*}{ Desconforto joelho direito } & Não & 13 & 6 & - & - & 0,289 \\
\hline & Sim & 2 & 0 & - & - & \\
\hline \multirow[t]{2}{*}{ Dor joelho direito } & Não & - & - & 17 & 1 & 1,000 \\
\hline & Sim & - & - & 2 & 1 & \\
\hline \multirow[t]{2}{*}{ Desconforto joelho esquerdo } & Não & 12 & 9 & - & - & 0,004 \\
\hline & Sim & 0 & 0 & - & - & \\
\hline \multirow{2}{*}{ Dor joelho esquerdo } & Não & - & - & 18 & 0 & 0,250 \\
\hline & Sim & - & - & 3 & 0 & \\
\hline \multirow[t]{2}{*}{ Desconforto perna direita } & Não & 13 & 3 & - & - & 0,625 \\
\hline & Sim & 1 & 4 & - & - & \\
\hline \multirow{2}{*}{ Dor perna direita } & Não & - & - & 16 & 0 & 0,250 \\
\hline & Sim & - & - & 3 & 2 & \\
\hline \multirow[t]{2}{*}{ Desconforto perna esquerda } & Não & 12 & 4 & - & - & 0,687 \\
\hline & Sim & 2 & 3 & - & - & \\
\hline
\end{tabular}




\begin{tabular}{|c|c|c|c|c|c|c|}
\hline \multirow[t]{2}{*}{ Dor perna esquerda } & Não & - & - & 16 & 1 & 0,625 \\
\hline & Sim & - & - & 3 & 1 & \\
\hline \multirow[t]{2}{*}{ Desconforto tornozelo direito } & Não & 15 & 4 & - & - & 0,125 \\
\hline & Sim & 0 & 2 & - & - & \\
\hline \multirow[t]{2}{*}{ Dor tornozelo direito } & Não & - & - & 17 & 0 & 0,250 \\
\hline & Sim & - & - & 3 & 1 & \\
\hline \multirow[t]{2}{*}{ Desconforto tornozelo esquerdo } & Não & 14 & 5 & - & - & 0,063 \\
\hline & Sim & 0 & 2 & - & - & \\
\hline \multirow[t]{2}{*}{ Dor tornozelo esquerdo } & Não & - & - & 19 & 0 & 0,500 \\
\hline & Sim & - & - & 2 & 0 & \\
\hline
\end{tabular}

Dados apresentados em frequência; * Teste de McNemar

\section{CONSIDERACÕES FINAIS}

Após a realização de um programa de ginástica laboral, foram observadas melhoras significativas na circunferência da cintura, relação cintura-quadril e flexibilidade. No entanto, não foram observadas alterações na percepção de desconforto e dor musculoesquelética nos trabalhadores.

\section{REFERÊNCIAS}

1. Candotti CT, Stroschein R, Noll M. Efeitos da ginástica laboral na dor nas costas e nos hábitos posturais adotados no ambiente de trabalho. Rev Bras Ciênc Esporte 2011; 33(3):699-714.

2. Pastre $\mathrm{E}$, Carvalho Filho G, Pastre $\mathrm{CM}$, Padovani $\mathrm{CR}$, Almeida JS, Netto Junior J. Work-related musculoskeletal complaints by women in a social rehabilitation center. Revista Cad Saúde Pública 2007;23(11):2605-12. doi: 10.1590/S0102$311 \times 2007001100008$

3. Gurgueira GP, Alexandre NMC, Corrêa Filho HR. Prevalência de sintomas músculo-esqueléticos em trabalhadoras de enfermagem. Rev Latino-Am Enfermagem 2003;11(5): 60813. doi: 10.1590/S0104-11692003000500007

4. Maciel MG. Ginástica Laboral, instrumento de produtividade e saúde. Barueri: Manole, 2008.

5. Zebis MK, Lars L Andersen LL, Pedersen MT, Mortensen $P$, Andersen $\mathrm{CH}$, Pedersen MM, Boysen $\mathrm{M}$, Roessler $\mathrm{KK}$, Hannerz H, Mortensen OS, Sjøgaard G. Implementation of neck/shoulder exercises for pain relief among industrial workers: A randomized controlled trial. BMC Musculoskeletal Disorders 2011;12(205):1-9. doi: 10.1186/1471-2474-12-205

6. Andersen LL, Andersen $\mathrm{CH}$, Mortensen OS, Poulsen OM, Bjornlund IB, Zebis MK. Muscle activation and perceived loading during rehabilitation exercises: comparison of dumbbells and elastic resistance. Phys Ther 2010;90(4):53849. doi: $10.2522 / p t j .20090167$

7. Figueiredo F, Mont'alvão C. Ginástica Laboral e Ergonomia. Rio de Janeiro: Editora Sprint, 2005.

8. Mendes RA, Leite N. Ginástica Laboral: Princípios e Aplicações Práticas. Barueri: Editora Manole, 2004.

9. Zilli CM. Ginástica Laboral: Uma tarefa interdisciplinar com ação multiprofissional. São Paulo: LOVISE, 2002.

10. Oliveira João Ricardo Gabriel de. A prática da ginástica laboral. Rio de Janeiro: Sprint, 2002.

11. Laux RC, Pagliari P, Effting Junior JV, Corazza ST. Programa de Ginástica Laboral e a Redução de Atestados Médicos. Cienc Trab 2016;18(56):130-133. doi: 10.4067/S071824492016000200009

12. WHO. World Health Organization. Obesity: preventing and managing the global epidemic. Report of a World Health Organization Consultation. Geneva: World Health Organization, 2000

13. Siri WE. Body composition from fluid spaces and density: analysis of methods. In: Brozek J, Henschel A, editors. Techniques for measuring body composition. Washington: National Academy of Sciences; 1961. p. 223-44.

14. Pollock MLE, Wilmore JH. Exercícios na saúde e na doença: avaliação e prescrição para prevenção e reabilitação. 2 ed. Rio de Janeiro: MEDSI; 1993.

15. Nahas Markus Vinicius. Atividade física, saúde e qualidade de vida. Londrina: Midiograf, 2001.

16. Castro CES. A formação linguística da dor - versão brasileira do questionário McGill de dor (Tese). São Carlos: Universidade Federal de São Carlos, 1999.

17. Moreira-Silva I, Santos R, Abreu S, Mota J. The effect of a physical activity program on decreasing physical disability indicated by musculoskeletal pain and related symptoms among workers: a pilot study. Int J Occup Saf Ergon 2014;20(1):55-64. doi: 10.1080/10803548.2014.11077028

18. Muyor JM, López-Miñarro PA, Casimiro AJ. Effect of stretching program in an industrial workplace on hamstring flexibility and sagittal spinal posture of adult women workers: a randomized controlled trial. J Back Musculoskelet Rehabil 2012;25(3):161-9. doi: 10.3233/BMR-2012-0323

19. Lee H, Chae D, Wilbur J, Miller A, Lee K, Jin H. Effects of a 12 week self-managed stretching program among KoreanChinese female migrant workers in Korea: a randomized trial. Jpn J Nurs Sci 2014;11(2):121-34. doi: 10.1111/jjns.12010

20. Santos AF, Oda JY, Nunes APM, Gonçalves L, Garnés FLS. Benefícios da ginástica laboral na prevenção dos distúrbios osteomusculares relacionados ao trabalho. Arq Ciênc Saúde Unipar 2007;11(2):107-13.

21. Sedrez JÁ, Rosa MIZ, Cunha A, Candotti CT. Avaliação dos efeitos de um programa de ginástica laboral, sobre a dor e a qualidade de vida. Cinergis 2012;13(2):21-6.

22. Silveira JWP, Laat EF, Pilatti LA, Silva F, Machowski CM, Samila G. Ginástica laboral e prevenção de dores crônicas. Cinergis 2013;14(2):115-9.

23. Ferreira KS, Santos AP. Os benefícios da ginástica laboral e os possíveis motivos da não implantação. Revista Educação Física UNIFAFIBE 2013;2(2):56-72. 